\title{
Yukawa Potential in Klein-Gordon Equation in Cosmological
}

\section{Inertial Frame}

\author{
Sangwha-Yi* \\ Department of Math, Taejon University 300-716, South Korea
}

*Corresponding Author: Sangwha-Yi, Department of Math, Taejon University 300-716, South Korea

Abstract: We study Yukawa potential dependent about time in cosmological inertial frame. If we solve KleinGordon equation, we obtain Yukawa potential dependent about time in cosmological inertial frame.

Keywords: Yukawa potential; Klein-Gordon equation; Cosmological inertial frame

PACS Number: 03.30. $+p, 03.65$

\section{INTRODUCTION}

Our article's aim is that we make Yukawa potential theory in cosmological inertial frame.

At first, Robertson-Walker metric is

$d \tau^{2}=d t^{2}-\frac{1}{c^{2}} \Omega^{2}(t)\left[\frac{d r^{2}}{1-k r^{2}}+r^{2} d \Omega^{2}\right]$

According to $\Lambda C D M$ model, our universe's $\mathrm{k}$ is zero. In this time, if $t_{0}$ is cosmological time[3],

$k=0, t=t_{0}>\Delta t, \Delta t$ is period of matter's motion

Hence, the proper time is in cosmological time,

$$
\begin{aligned}
& d \tau^{2}=d t^{2}-\frac{1}{c^{2}} \Omega^{2}\left(t_{0}\right)\left[d r^{2}+r^{2} d \Omega^{2}\right] \\
& =d t^{2}-\frac{1}{c^{2}} \Omega^{2}\left(t_{0}\right)\left[d x^{2}+d y^{2}+d z^{2}\right] \\
& =d t^{2}\left(1-\frac{1}{c^{2}} \Omega^{2}\left(t_{0} V^{2}\right), \quad V^{2}=\frac{d x^{2}+d y^{2}+d z^{2}}{d t^{2}}\right.
\end{aligned}
$$

In this time,

$$
d \bar{t}=d t, d \bar{x}=\Omega\left(t_{0}\right) d x, d \bar{y}=\Omega\left(t_{0}\right) d y, d \bar{z}=\Omega\left(t_{0}\right) d z
$$

Cosmological special theory of relativity's coordinate transformations are

$$
\begin{aligned}
& c \bar{t}=c t=\gamma\left(c \bar{t}+\frac{V_{0}}{c} \Omega\left(t_{0}\right) \bar{x}^{\prime}\right)=\gamma\left(c t+\frac{V_{0}}{c} \Omega\left(t_{0}\right) x ' \Omega\left(t_{0}\right)\right) \\
& \bar{x}=x \Omega\left(t_{0}\right)=\gamma\left(\bar{x}+v_{0} \Omega\left(t_{0}\right) \bar{t}^{\prime}\right)=\gamma\left(\Omega\left(t_{0}\right) x+v_{0} \Omega\left(t_{0}\right) t^{\prime}\right)
\end{aligned}
$$




$$
\begin{aligned}
& \bar{y}=\Omega\left(t_{0}\right) y=\bar{y}^{\prime}=\Omega\left(t_{0}\right) y^{\prime}, \quad, \gamma=1 / \sqrt{1-\frac{v_{0}^{2}}{c^{2}} \Omega^{2}\left(t_{0}\right)} \\
& \bar{z}=\Omega\left(t_{0}\right) z=\bar{z}^{\prime}=\Omega\left(t_{0}\right) z^{\prime}
\end{aligned}
$$

\section{Yukawa Potential in Klein-Gordon Equation in Cosmological Inertial Frame}

If we focus Klein-Gordon equation about Yukawa potential $\phi$ dependent about time,

$\frac{m_{\pi}{ }^{2} C^{2}}{\hbar^{2}} \phi+\partial_{\mu} \partial^{\mu} \phi=\frac{m_{\pi}{ }^{2} C^{2}}{\hbar^{2}} \phi+\frac{1}{C^{2}} \frac{\partial^{2}}{\partial t^{2}} \phi-\nabla^{2} \phi=0$

In this time, Yukawa potential $\phi$ dependent about time is.

$\phi=-\frac{g^{2}}{r} \exp \left(-\frac{m_{\pi} r C}{\hbar}\right)+A_{0} \sin \omega t$

Frequency $\omega=\frac{m_{\pi} C^{2}}{\hbar}, m_{\pi}$ is meson's mass

$\mathrm{Eq}(6)-K l e i n-G o r d o n$ equation is satisfied by $\mathrm{Eq}(7)-Y u k a w a$ potential dependent about time In cosmological inertial frame, Klein-Gordon equation is[2]

$$
-\Omega\left(t_{0}\right) \frac{1}{C^{2}} \frac{\partial^{2} \phi^{\prime}}{\partial t^{2}}+\frac{1}{\Omega\left(t_{0}\right)} \nabla^{2} \phi^{\prime}=\frac{m_{\pi}^{2} C^{2}}{\hbar^{2}} \phi^{\prime}
$$

In this point, in cosmological inertial frame, space-time transformations in the type A of wave function and the other type B of the expanded distance are

Type A: $r \rightarrow r \sqrt{\Omega\left(t_{0}\right)}, t \rightarrow \frac{t}{\sqrt{\Omega\left(t_{0}\right)}}$, Type B: $r \rightarrow r \Omega\left(t_{0}\right), t \rightarrow t$

Space-time transformation of Yukawa potential $\phi^{\prime}$ is depend on Type A

Hence, Yukawa potential $\phi^{\prime}$ dependent about time is

$\phi^{\prime}=-\frac{g^{2}}{r \sqrt{\Omega\left(t_{0}\right)}} \exp \left[-\frac{m_{\pi} r \sqrt{\Omega\left(t_{0}\right)} c}{\hbar}\right]+A_{0} \sin \left(\frac{\omega t}{\sqrt{\Omega\left(t_{0}\right)}}\right)$

Frequency $\omega=\frac{m_{\pi} c^{2}}{\hbar}, m_{\pi}$ is meson's mass

$\mathrm{Eq}(8)-\mathrm{Klein}$-Gordon equation is satisfied by $\mathrm{Eq}(10)$-the solution.

\section{CONCLUSION}

We solve Klein-Gordon equation in cosmological inertial frame. Hence, we found Yukawa potential dependent time in cosmological inertial frame.

\section{REFERENCES}

[1]S.Yi, "Vibration of Yukawa Potential Depentent Time and Extended Klein-Gordon Equation in Rindler Space-Time”, International Journal of Advanced Research in Physical Science,7,7(2020),pp1-4

[2]S.Yi, "Klein-Gordon Equation and Wave Function in Cosmological Special Theory of Relativity", International Journal of Advanced Research in Physical Science,7,12(2020),pp4-6

[3]S.Yi, "Cosmological Special Theory of Relativity", International Journal of Advanced Research in Physical 
Science, 7,11(2020),pp4-9

[4]J.D. Bjorken \& S. D. Drell, Relativistic Quantum Field(McGraw- Hill Co., 1965)

[5]P.Bergman,Introduction to the Theory of Relativity(Dover Pub. Co.,Inc., New York,1976), Chapter V

[6]R.L.Liboff, Quantum Mechanics(Addison-Wesley Publishing Co., Inc., 1990)

[7]A.Beiser, Concept of Modern Physics(McGraw-Hill,Inc.,1991)

[8]H. Yukawa, "On the interaction of elementary particle. I", Proceedings of the Physico-Mathematical Society of Japan. $3^{\text {rd }}$ Series 17(1935):48-573

Citation: Sangwha-Yi (2021). Yukawa Potential in Klein-Gordon Equation in Cosmological Inertial Frame. International Journal of Advanced Research in Physical Science (IJARPS) 8(3), pp.16-18, 2021.

Copyright: (C) 2021 Authors, This is an open-access article distributed under the terms of the Creative Commons Attribution License, which permits unrestricted use, distribution, and reproduction in any medium, provided the original author and source are credited. 